\section{Beitrag zur modernen Diagnostik der Magenkrankheiten.}

Von Dr. med. Hermann Lenhartz, Priv.-Docenten für innere Medicin in Leipzig.

(Schluss aus No. 6.)

Bei unseren Fällen von Chlorose mit Dyspepsie fanden wir - wie oben bemerkt - in fast der Hälfte verminderte Saftsecretion. Ritter und Hirsch waren wohl die ersten, die bei Chlorose und Anämie Verminderulg der Salzsäure beobachteten. Aber auch Müller constatirte bei weiblichen chlorotischen Ulcuskranken der Gerhardt'schen-Klinik bisweilen nentralen Mageninhalt. Meiner $A$ nsicht nach kann dieser von verschiedenen Seiten erhobene Befund nicht auffällig erscheinen. Bei jeder schweren Chlorose überzeugen wir uns, dass alle Functionen des Körpers mehr oder weniger schwer gestört sind. Herzklopfen, Athemnoth, Appetitmangel, träge Verdauung, Fehlen der Menses, rasch eintretende körperliche und geistige Ermüdung sind stetig wiederkehrende Erscheinungen. Dürfen wir uns da wundern, dass die Secretion der starken Säure, deren Abscheidung von Bunge ${ }^{1}$ ) mit Recht als eine bemerkenswerthe Leistung der lebenden Drüsenzelle bezeichnet wird, eine Verminderung erfährt?

Wenn wir nun aber der verlockenden Erklärung, dass der die Chlorose begleitenden Hyperacidität bei der Entstehung des peptischen Magengeschwürs eine entscheidende Rolle zufallen soll, nur fïr gewisse Fälle einen Platz einräumen können, wie dürfen wir uns denn die Beziehungen zwischen den beiden offenbar häufig zusanmentreffenden Krankheitszuständen vorstellen? Mir scheint folgende Ueberlegung wohl statthaft. Nicht selten beobachten wir bei blutarmen Personen, zumal bei chlorotischen Franen, eine auffällige Neigung zn Blutnngen aus der Nase, bezw. wenn die Menses nicht cessiren, relativ häufig eine übermässige Menstrualblutung. Wir können ferner feststellen, dass das Nasenbluten sehr häufig auf geringfügige meclianische Reize, wie stärkeres Schnaufen u. dergl. erfolgt. Da dürfen wir, meiner Ansicht nach, anch von Seiten der Magenschleimhant rine ähnliche Empfindlichkeit voraussetzen, und zwar um so mehr, wenn entweder infolge von Hyperacidität ein krampfhafter Verschluss des Pylorus (Riegel) die Speisen länger im Magen zurückhält und die Verdauung der Amylaceen, besonders der Brodkrnsten u. dergl., verzögert, oder durch die mangelhafte Saftabsonderung die Auflösung der lıgesta aufgehalten wird. Hier wird - zumal bei dem Ausfall der antiseptischen Wirkung der Salzsänre, die Bildung organischer Säuren begünstigt und bacteriellen Einflïssen ungenügend entgegengewirkt. ${ }^{2}$ ) Kommt es dann infolge chemischer oder mechanischer Einflüsse zn einer auch nur geringfügigen Läsion der entschieden weniger widerstandsfähigen Schleimhaut, so wird sowohl der normale als unternormale HCl-Gehalt zumal bei Mitwirkuug der organischen Säuren - genïgen, um clie Heilung des oberflächlichen Substanzverlustes zn verzögern und Ausbildung eines Ulcns beizutragen. Ich muss aber Ewald's Satz, ndass die veränderte Blutbescliaffenheit auch bei normaler (bezw. wie ich meine auch unternormaler) Acidität als disponirendes Moment zu gelten hat", auch aus einem anderen Grunde beipflichten; denn meiner Ansicht nach mïssen wir die Blutveränderung mit dafür verantwortlich machen, dass jene Defecte so verhältnissmässig oft nicht verheilen. Nnr so können wir uns das relativ häufige Vorkommen von Ulcus ventr. bei Blutarmen erklären, da wir bei anderen Kranken selbst nach wiederholten kleinen Blutungen und Epithelabstossungen ein Ulcus sich nicht entwickeln selien.

Mit grossem Interesse habe ich ferner die Erscheinungen beachtet, wie sie bei Magenerweiterung durch die Expressionsmethode klargelegt wurden. Bei der oben angegebenen Krnnkenziffer habe ich 16 Fälle von melır oder weniger hochgradiger Erweiterung verfolgt. Bei 2 Kranken konnte nit aller Sicherheit eine carcinomatöse Strictur des Pylorus angenommen werden; hier fehlte bei wiederholten Aushełerungen sowohl nach Milchfrühstïck, als gemischter Kost freie $\mathrm{HCl}$ durchweg. (Ein Fall durch Autopsie bestätigt.)

Von 5 Kranken, bei welchen mit Bestimmtheit früher Ulcus ventr. vorausgegangen war, beziiglich noch bestand, boten nur 2 ïbernormalen $\mathrm{HCl}$ (Werthe von $0,26-0,290 \%$ nach Probefrühstïck) dar, während freie $\mathrm{HCl}$ bei dem dritten nur zu 0,13-0,18\% nach Probemahlzeit gefunden, und bei den beiden letzten regelmässiges Fehlen beobachtet wurde; nur einmal konnten wir, nachdem wir kurz nach einer Ausspülung Wasser in den Magen irrigirt

1) Lehrb. der physiol. und pathol. Chemie 1887, 9. Vorlesung.

2) In jüngster Zeit hat Kast (Ueber die quantitative Bemessung der antiseptischen Leistung des Magensaftes; Festschrift, Hamburg 1889) in überzeugender Weise den bedeutsamen hemmenden Einfluss der freien $\mathrm{HCl}$ auf die Darmfäulniss erwiesen. hatten, etwa $3 / 4$ Stunden später einen schwachen HCl-Werth feststellen.

Vier weitere Fälle, bei denen kein Anlass zur Diagnose eines Ulcus vorlag, ergaben bei wiederholten Untersuchungen lebhafte Reaction freier $\mathrm{HCl}$, so dass es in der That nahe lag, an den von Riegel u. a. beschuldigten krampfhaften Verschluss des Pylorus zn denken. Indess sprach doch bei 2 dieser Kranken der Umstand gegen diese Annahme, dass auf Milchfrühstiick nur ein Gehalt von $0,16-0,18 \%$ fr. $\mathrm{HCl}$ gefunden wurde.

Ich habe soeben bereits 2 Kranke erwähnt, bei denen, obschon Ulcus vorausgegangen war, freie $\mathrm{HCl}$ in der Regel fehlte. Ausser diesen aber eroab bei $\tilde{5}$ weiteren Kranken die Untersuchung stetes Fehlen freier Salzsäure. ${ }^{1}$ ) $O b$ in solchen Fällen in der That, wie Klemperer ${ }^{2}$ ) u. a. vermuthen, ein Stadium von Hyperacidität vorausgeht, das der Anacidität weicht, kann nach meinem Dafürhalten nur durch directe längere Beobachtıng bei einem und lemselben Kranken ermittelt werden. Jedenfalls möchte ich nach meinen Untersuchungen in der Schwäche der motorischen Magenfunction die hauptsächlichste Ursache für solche Fälle von Magenerweiterung suchen, bei denen dieselbe ohne vorausgegangenes Ulcus (bezw. Carcinoma pylori) sich entwickelt.

Ausdrücklich sei noch bemerkt, dass die Diagnose der Dilatatio ventr. nur dann gestellt wurde, wenn wiederholte Eingiessungen bezw. die Aufblähung durch $\mathrm{CO}_{2}$ die grosse Curvatur in Nabelhöhe oder mehrere Finger breit unter derselben erscheinen liessen, und nebenher mehr oder weniger lebhafte peristaltische Bewegungen die Diagnose stützten.

Auf eine genauere Prïfung der motorischen Kraft, zumal nach der Vorschrift von Klemperer, konnte ich mich bei meinem Krankenmaterial nicht einlassen. Ich möchte aber - obwohl icl jene Methode niemals ausgefiihrt habe - gewisse Bedenken bezüglich der Zuverlässigkeit derselben nicht unterdrücken. Anf Grund der reichen Erfahrnngen, die ich sowohl früher an der hiesigen medicinischen Klinik, als in meiner Poliklinik gesamınelt habe muss ich diese Zweifel äussern. Wie oft wird man gerade bei der Ausspülung eines dilatirten Magens durch das Auftreten mannichfacher Speisereste noch überrascht, nachdem - sei es durch einfache Heberwirkung, sei es durch die Magenpumpe - eine mehr oder weniger grosse Menge klaren Spilwassers längere Zeit hindurch entleert ist. Sollte es da nicht möglich sein, dass eine gewisse Menge des von Klemperer eingefïhrten Oels ab und zu im Magen zurïckbleibt, und das Fehlen dieses Quantums irrthümlich eine gute motorische Leistung voraussetzen lässt?

Wenn Klemperer diesem Einwand gegenüber geltend macht dass , viele Versuche, die er znerst an Hunden, einige Mal auch an Menschen gemacht habe, dem widersprächen, da er bei der auf die Eingiessung alsbald folgenden Aspiration das Oel ohne Verlust wieder erhalten hätte, " so erscheint mir diese Vertheidigung nicht stichlialtig. Einmal hat es sich gerade bei diesen Versuchsobjecten um gesunde Mägen gehandelt, zum andern ist die Entnahme unmittelbar der Einführung des Oels gefolgt. Es liegen somit die Verhältnisse bei diesen Controlversuchen doch anders, als bei Kranken mit Magenerweiternng. Hier haben wir es nicht selten mit einem schlaffen Sack zu thun, der bisweilen verschiedene Ausbuchtungen darbietet. Und wenn wir dann beobachten, dass es sich selbst nach der Durchspülung von einigen Litern Wasser immer noch ereignen kann, dass später mit Nahrungsresten vermischtes Wasser - plötzlich - auftritt, so ist wohl der Schluss erlaubt, class auch vom Oel ein Rest zurückbleiben kann, der nicht durch Aspiration zu gewinnen ist.

Bei der Analyse meiner Beobachtungen habe ich schon an mehreren Stellen auf die Abhängigkeit gewisser Magenstörungen von nervösen Zuständen hingewiesen. Schon bei den acuten Dyspepsieen musste ich zur Erklärung der hin und wieder zu beobachtenden Hyperacidität nervöse Einflüsse in Anspruch nehmen; gallz besonders aber bei den chronischen Formen der übermässigen Saftsecretion. Ich glaube aber auch mit grösster Wahrscheinlichkeit für manche Formen von Anacidität gewisse Nervenstörungen beschuldigen zn müssen. Anders möchte ich wenigstens nicht die Beobachtung erklären, dass bei 3 Schwangeren (von denen eine, im 4. Monat gravida, seit 8 Wochen an Hyperemesis litt) absolutes Fehlen freier $\mathrm{HCl}$ beobachtet wurcle; so möchte ich es auch auffassen, wenn bei 8 Frauen mit Ren mobilis freie $\mathrm{HCl} 3$ mal nahezu, 5 mal völlig fehlte.

Im übrigen will ich nur kurz bemerken, dass ich bei ansgesprochenen Neurasthenikern, die über mehr oder weniger starke Magenbeschwerden klagten, und zwar bei 13 Männern und 12 Frauen die Reaction auf freie $\mathrm{HCl}$

1) Zum Theil habe ich über die Fälle von Ectasie auf dem Congress für innere Medicin berichtet; s. die Verhandlungen 1889, p. 286.

2) Ueber die Magenerweiterung und ihre Behandlung. Ibidem p. 271 u. ff. 
bei 3 Männern und 2 Frauen zu stark $(0,3-0,36-0,5$ p. c. $)$ $\Rightarrow 4 \pi, \quad \pi \quad n$ normal,

$72 \pi 3 \%$ zu schwach,

"Hier muss man"also auf ein sehr verschiedenes Verhalten des Chemismus gefasst sein.

Nur flüchtig will ich noch erwähnen, dass ich in eivem Falle von chronischem Erbrechen, das ich wohl mit Recht als urämische Erscheinung deute, weil der betr. Herr seit etwa 15 Jahren an chronischer Cystopyelitis leidet, stets reichen Gehalt an freier $\mathrm{HCl}$ fand, dass in einem $\mathrm{z}$ weiten Falle von sicherem urämischen $\mathrm{Er}$ brechen bei einer chronischen Nierenkranken dagegen regelmässiges Fehlen der freien $\mathrm{HCl}$ beobachtet wurde. Auch in einem Falle von Addis on'scher Krankheit, der bei der Autopsie typische tuherculöse Erkrankung der Nebennieren ergab, fehlte freie Säure.

Von Interesse dürften ferner noch einige Bemerkungen sein, die sich auf den Chemismus solcher Magenkranken beziehen, bei denen ausgesprochenes Potatorium vorlag. Hier fand ich bei einem Kranken die Secretion normal, bei einern anderen Fall Hyperaci dität (6 Express.), bei 6 weiteren Fällen Anacidität $(\mathrm{HCl})$.

Mit wenigen Worten sei \%um Schluss noch des gleichzeitigen Vorkommens von bew glicher Niere und Dyspepsie gedacht. In 19 Fällen, und zwar bei 17 Frauen und 2 Männern, fiude ich diese Coinciden\% verzeichnet. Ich hebe in erster Linie als bemerkenswerth hervor, dass ich in meinen Fällen von Magenerweiterung nicht ein einziges Mal gleichzeitige bewegliche Niere feststellen konnte, obwohl die bimanuelle Untersuchung und andere Vorschriften nie versäumt wurden. Ich habe ferner srhon erwähnt, dass ich bei 5 Fällen Fehlen, bei 3 der Kranken heträchtliche Verminderung der Saftsecretion gefunden habe, und dass ich in diesen Fällen geneigt bin reflectorische Beziehungen anzunehmen. Hierfür spricht 11. a. die Wahruehmung, dass bei 5 weiteren Kranken der Befund wechselte, und bei je 3 Fällen normale bez. gesteigerte Secretion festgestellt wurde. Obwohl ich also bei den gemeinschaftlichen Vorkommen die Störung vermisse, auf die früher von Bartels und neuerdings erst wieder von Litten ${ }^{t}$ ) aufmerksam gemacht ist, halte ich es doch für angezeigt, in jedem Falle von chronischer Dyspepsie auf Ren mobilis $z u$ achten. Dass eine gewisse Abhängigkeit zwischen Dyspepsie und Beweglichkeit der Niere besteht, beweist auch mir die Erfahrung, dass die Magenbeschwerden nicht selten sofort durch das Tragen einer gut angepassten Leibbinde aufgehoben wurden.

Bei der Darstellung meiner Untersuchungen habe ich bisher fast ausschliesslich das Verhalten der freien $\mathrm{HCl}$ bei den verschicdenen Krankheitsgruppen berücksichtigt. Ich muss es nir versagen, über das Auftreten der Milchsäure und sonstigen organischen Säuren eingehender zu berichten; bei der grossen Zahl meiner poliklinischen Kranken ${ }^{2}$ ) war es mir nicht nöglich, jedem einzelnen Magenpatienten soviel. Zcit zu widmen, wie zur Feststellung und $\Lambda$ ufzeichnung des Verhaltens jener Säureu nöthig ist; meine Notizen sind in dieser Richtung lïckenhaft. Nur soviel darf ich erwälnen, dass ich in ziemlich zahlreichen Fällen auch dann, wenn die G ünzburg'sche Reaction positiv (ja stark ausfiel) einen lebhaften Ausschlag mit Eisenchloridcarbollösung beobachtet habe. Flïchtige Fettsäuren u. s. f. fand ich besonders stark in den Fällen, wo freie $\mathrm{HCl}$ fehlte, aber auch in solchen, bei denen die Saftsecretion normal (oder selbst gesteigert) war, kameı dieselben zur Wahrnehmung.3)

Die von mir befolgte Methode erlaubt aber auch ausser der Beurtheilung der in erster Limie klargelegten secretorischen Verhältnisse einen Rückschluss auf die motorischen und resorbirenden Vorgänge. Leider kann ich auch hier nicht auf eine genaue Darstellung eingehen, da meine Beobachtungen in dieser Beziehung ebenfalls unvollständig bleiben mussten. Dass ich in vielen Fällen, bei denen die freie Mineralsäure fehlte, oft noch naclı Stunden den Magen gefüllt fand, nnd die Reichlichkeit des Inhalts direct auf eine motorische (bezw. auch Resorptions-) Störung hilıwies, kanu nicht auffällig erscheineu.

Viel bemerkenswerther war für mich die in zahlreichen Fällen anzustellende Beobachtung, dass Kranke, bei denen ich durch wiederholte Prüfung zu verschiedenen Stunden der Verdauung eimen mehr oder weniger gestörten Chemismus festgestellt hatte, nuch nach reichlichen Probemahlzeiten nur noch wenig Chymus (31/2-4 Stundeu nach der Nahrumgsaufnahme) der Expression darboten. Ich konnte also die von anderer Seite hervorgehobene That-

1) Ueber den Zusammenhang von Erkrankungen des Magens mit Lageveränderungen der rechten Niere. Congr. f. innere Med. 1887, p. 223 u. ff.

2) Die von mir 1886 gegründete Poliklinik wurde im 1. Jahr von 847 , im 2. Jahr von 978, im 3. Jahr von 1342, in diesem erst Mitte April ablaufenden 4. Jahr bis jetzt von 1100 Kranken aufgesucht.

3) Auch die Peptonbestimmungen konnten aus demselben Grunde nicht regelmässig ausgeführt werden. sache bestätigen, dass bei secretorischer Störung die motorische Function compensatorisch eintreten kann. Indessen muss ich es für unrichtig erklären, wenn auf Grund solcher Beobachtungen der Mechanismus der Magenverdauung als die hauptsächlichste Function dargestellt wird. ${ }^{1)}$

Auch hier ist Ewald's Warnung am Plat\%, dass es gefährlich ist, aus klinischen Beobachtungen, selbst wenn sie wiederholt in gleichen Sinne aus\%uschlagen scheinen, allgemein gültige Regeln ableiten zu wollen." Ich habe schon oben bemerkt, dass ich in festen Glauben an die Sätze der Riegel'schen Sclule im Beginn meiner Untersuchungen gewisce Schlüsse gezogen habe, die einer kritischen Nachprüfung nicht Stand gehalten hätten. Bald merkte ich aber, dass die von jener Schule aus ihrem thatsächlichen Material erhobenen ["olgerungen für meine Kranken nur eine bedingte Gültigkeit beanspruchen durften. Dass dies so ist, bedauere ich selbst am allermeisten; denn statt der scheinbar einfachen Sachlage haben wir, trot\% der durch die Expression geförderten Diagnose, immer noch mit complicirten Factoren zu rechnen.

Die im Begiun meiner Arbeit aufgeworfene Frage, ob die diagnostische Anwendung des Magenrohrs unter Benutzung der von Leube, Riegel und Ewald gegebenen Vorschriften in der Praxis ausführbar sei, lahe ich oben schon kurz bejaht. Ich möchte aber nicht unterlassen, darauf aufmerksam zu machen, dass die Methode durchaus ungefährlich ist. Bei den 900 Expressionen und den angeschlossenen (mehreren 100) Ausspülungen habe ich nur zwei mal infolge der Expression Abstossung kleinster Schleimlıautpartikel und bei Ausspülungen nur in etwa 5-8 Fällen leicht blutige Verfärbung des Spülwassers beobachtet. Bei keinem der Fälle ist die geringste suhjective oder objective Störung danach aufgetreten. In den beiden Fällen von Schleimhautablösung hat die später nach acht Tagen wiederholte Expression keiue solche Folge gehabt. Einmal int es mir begegnet, dass bei der Ausspülung eiues Kranken mit Magenerweiterung etwas dunkles Blut ansfloss; hier gab der Kranke auf Befragen nachträglich an, dass der Stuhl an demselben Tage dunkel gewesen sei. Da bei dem Kranken starke Stagnation vorlag, und das Blut im Spülwasser chocoladenartig war, nahm ich an, dass die Blutung schou einige Tage alt sei. Der Kranke fühlte sich sofort nach der Aussü̈lung wohl, eine neue Blutung blieb aus.

Nach allem muss ich Ewald \%ustimmen, wenn er seine Methode als durchaus ungefïlırlich bezeichnet; indess halte ich dem verdienten Forscher gegenïber zwei nebensächliche Ausstellungen am Plat\%. Wenn derselbe erklïrt, dass ein hohes Maass von Ungeschicklichkeit dazu gehöre, wenn die Sonde in den Larynx geführt werde, so täuscht er sich. Obwoll ich im Besitz einer recht guten Dexterität bin, habe ich doch die Beobachtung gemacht, dass ich bei ein und demselbeu Kranken mit dem weichen Rohre regelmässig in den Kelılkopf kam und mit dem starren Rohr nur dann diesen Weg vermeiden konnte, wenn ich mit dem Finger den Kehlkopf schloss. Hier lagen also Verhältnisse vor, denen gegenüber mit dem weichen Rohre nichts auszurichten ist. Dass der $\Lambda \mathrm{n}$ fänger sehr häufig in den oberen Kehlkopf gelangt, beobachte ich alle Wochen in meiner Poliklinik, in der ich einen grossen Theil der Expressionen von den Praktikanten ausführen lasse. In solchen Fällen hört man ein lautes zischendes In- und Exspirationsgeräusch durch das Rohr hindurchgehen. Die Cyanose ist deshalb auch nicht so stark, wie in solchen Fällen, bei denen das Rohr schon das Ringknorpelhinderniss passirt lat, der Kranke aber zu athmen aufhört. Zur Vermeidung dieses (letzteren) scheinbar beängstigenden Verhaltens nuss man die Leute auffordern tief zu athmen; der erste Athem\%ug befreit sie von ihrer Angst.

Wenn Ewald weiter die Einführung des Rohres weniger belästigend nennt, als die Untersuchung mit dem Kehlkopfspiegel", so muss ich ilım entgeguen de gustibus non est disputandum". Ungemütllicher ist die Express'on doch auf alle Fälle. Ich stimme ihm bei, dass man nur selten eine Cocainpinselung nöthig hat ich habe nur in einem ein $\mathrm{ige}$ e Falle bei einer reizbaren jungen Dame diese Maassualıme nöthig gehabt und dann die vorher nicht ausführbare Expression erreicht.

Mir selbst hat endlich auch nur ein poliklinischer Patient (ein russischer Jude) die Expression verweigert, und die $\%$ wei anderen Kranken, die „darauf verzichteten", trauten meinem Famulus nicht recht. All dies beweist, dass die Methode in der Praxis sebr wohl ausführbar ist.

Nicht so einfach erscheint mir die Beantwortung der Frage, ob diese Untersuchungsmethode nothwendig ist. Icl zweifle nicht daran, dass man in der Privatpraxis sehr häufig ohne dieselbe auskommen kann, dass man häufig durch diätetische Vorschriften u. dergl. eine Besserung erzielt, ohne eine exacte Diagnose gestellt zu haben; das habe ich selbst oft genug erlebt. Aber darüber darf man sich meiner Ansicht nach nicht täuschen, dass

1) S. Grundzach, Berl. klin. Wochenschr. 1887, No. 30. 
IIın ohne jene Untersuchung häufig im Dunke!n tappt. Und hier sei es mir gestattet die Frage zn erörtern, ob wir denn durch die von den verschiedensten Seiten gewonnenen Expressionsbefunde noch nicht zu dem wïnschenswerthen Ergebniss gekommen sind, diesen oder jenen Symptomencomplex mit einem bestimmten, bei den Expressionen in der Regel wiederkehrenden Befund in Einklang $\iota u$ bringen, so zwar, dass die Expression unterlassen werden könne. Ich fürcbte, dass dies Beginnen (obwohl von mancher Seite schon versucht1) zunächst $110 \mathrm{ch}$ als verfrüht $z u$ erachten ist.

Nur einige Zeichen scheinen mir schon jetzt verwerthbar zu sein. Einschliesslich der acuten Dyspepsieen, bei denell, wie wir gesehen haben, in der übergrossen Mehrzahl der Fälle die Saftsecretion vermindert und dementsprechend meistens von vornlierein Salzsäurezufuhr zu rathen ist, beobachten wir in der Regel eine mehr oder weniger belegte Zunge, Appetitmangel, Widerwillen gegen das Essen, besonders bei den mit verminderter Secretion verlaufendell Störungen. Wir finden ein tadelloses Verhalten der Zunge und lı̈ıfig sicb einstellendes Hungergefïhl bei Kranken mit Hyperacidität; wir erfahren endlich, dass bei dieser Gruppe in der Regel Verstopfung besteht, während der Stıhl bei jenen Kranken nicht selten regrelmässig ist. Aber daniit sind wir anch fast am Ende. Wenn heftiger Spontan- und Druckschmer\% als regelmässiges Symptom von Hyperacidität angeführt wird, so muss ich denı durchaus widersprechen. In zahlreichen Fällen, in denen ich selbst Anamnese und Status genau aufnahm ind ans dem Druckschmerz und anderen Symptomen auf secretorische Steigerung gefasst sein musste, ergab die Auspressung das gerade Gegentheil. Diese Fälle sind so häufig, dass man sie nicht als Ausnahme ansehen darf, und icl habe gerade sie dazu benutzt, um meinen Praktikanten immer auf's neue den Werth der Ausheberungen vorzufülıren. Nur den Fällen, bei denen der Druckschmerz eine andere Stelle als die Regio pylorica betrifft, be\%. intensive Schmerzen an einer bestimmten Stelle der vorderen Wand oder von Magenleidenden heftige Rückenschmerzen geklagt werden, möchte ich fïr die Diagnose des Ulc. rotund. einen Werth beimessen. Dagegen habe ich - entgegen manchen Angaben - 3 Fälle von durch die Autopsie bestätigtem Carcinoma ventriculi beobachtet, bei denen die über Monate sich hinziehende regelmässige Gewichtszunahme an der Diagnose zweifeln liess, bis der Exitus letalis die anfängliche Annahme bestätigte.

Dass ferner Anämie und Chlorose durchaus nicht zur. Annahme einer Hyperacidität berechtigen, dass Erbrechell auch bei zahlreichen Kranken mit verminderter Secretion auftritt, und dass man bei hochgradiger Nervosität auf die verschiedensten Ergebnisse gefasst sein muss, endlich ausstrahlende Schmerzen sowohl bei Kranken mit gesteigerter, als mangelhafter Saftabsonderung beobachtet werden, sind so häufige Beobachtungen, dass meine Bedenken Jaworski, Boas u. a. gegenüber, die jene Symptome als charakteristisch aufrestellt haben, gerechtfertigt siud.

Wenn über die Nothwendigkeit der Expression Bedenken erlaubt sind, so iniissen dieselben verstuminen, wenn die grössere Sicherheit des therapeutischen Handelns in Frage kom mt. Denu darüber kann kein Zweifel obwalten, dass die Diagnose infolge der Ausheberung an Sicherlieit gewinnt. Ich bin weit davon entferıt, in den Schematisinus zu gerathen und Magenkranke in solche zu trennen, bei denen Säure fehlt oder vorhanden ist, be\%. übermässig abgesondert wird, und für die Fälle mit gesteigerter Secretion neutralisirende Mittel, für die erstere Gruppe Säure \%u verordnen. Wohl wende ich in jedem Falle, bei dem freie $\mathrm{HCl}$ im Filtrat fehlt, verdünnte Salzsäure an und schwanke in den Gaben $z$ wischen 4-10 $\mathrm{Tr}$. nach einer kleinen, und 8-12-20 Tr. nach einer grösseren Mahlzeit und lasse dieselben auf $1 / 2-1$ Wasserglas roll $1-1 \frac{1}{2}$ Stunden nach dem Essen schluckweise nehnen. Nie habe ich nachtheilige Folgen beobachtet sondern, von vereinzelten Ausnabmen abgesehen, bei denen die Erleichterung ausblieb, stets den bestell Nut\%en wahrgenommen. Wenn fast 200 Kranke nach einem Mittel subjective und objective Besserung darbieten, wenn das Gefühl vou Druck und Völle und das Erbrechen darnach aufhören, der Appetit sich regt u. s. f, so dürfen wir diese Erscheinungen wohl direkt auf das Mittel beziehen. $\mathrm{Ob}$ der gïnstige Einfluss in erster Linie der antiseptischen Wirkung zukommt, oder zugleich eine Anregung der Drüsenthätigkeit mitspielt, bez. durch sie die Bildung der für die Peptonisirung nöthigen sauren Albuminate ermöglicht und gefördert wird, wage ich zunächst nicht zu entscheiden.

Dass fïr Kranke mit Hyperacidität allgemeine diätetische Rathschläge nicht viel nützen, ist schon oft hervorgeboben. Gerade hier schafft man die grösste Erleichterung durch die den Secretionsverhältnissen angepasste Empfehlung reichlicher Eiweisskost u. s. f.,

1) Jaworski, Subjective Magensymptome - objectiver Befund; Wiener med. Wochenschr. 1886, No. 49-52 und Boas, Zur Symptomatologie des chronischen Magenkatarrhs u. s. w.; Nünch. nned. Woehenschr. 1887, No. $41-42$. nöthigenfalls durch regelmässige Ausspülungen, fïr deren systematische Einführung wir Kussmaul grossen Dank schulden.

Einen Hauplwerth bietet die Ausheberung noch dadurch, dass man bei Feststellung ausgedehnter Zersetzungen u. dergl. die Ausspülung des Magens sofort anschliessen wird. Dass diese Maassnahne nicht nur bei den Erweiterungen, sondern sowohl bei acuten als chronischen Störungen das beste leistet, hat mir jeder neue Fall bewiesen.

Meine Arbeit bezweckte, das Ergebuiss der an einem ziemlich grossen Material nacb einheitlicher Methode gewonnenen Erfahrungen bekannt zu geben und vor allem der, meines Erachtens nach, in der Praxis nicht genïgend gewïrdigten diagnostischen Ausheberung des Magens Auhänger zuzuführen. Es sollte mich freuen, wenn meine Mittheilungen zur weiteren Ansbreitung der wichtigen Untersuchungsmethode anregen würden. 